\title{
Low-Dose Intravenous Ketamine Bolus versus Conventional Technique for the Reduction of Upper and Lower Extremity Fractures in Children: A Randomised Controlled Clinical Trial
}

\author{
(D) Mehdi Nasr Isfahani, (D) Narges Shahverdi, (D) Keihan Golshani
}

Department of Emergency Medicine, Isfahan University of Medical Sciences, Isfahan, Iran

\begin{abstract}
Aim: Ketamine administration in high dosage leads to certain adverse reactions. This study aimed to evaluate the effect of low-dose intravenous ketamine bolus versus conventional injection for the reduction of upper and lower extremity fractures in children.

Materials and Methods: A total of 198 paediatric patients with extremity fractures were enrolled. They were randomly categorized into two groups. In the intervention group, a number of 100 patients participated with a mean age of $8.89 \pm 4.37$, among them 77 (77\%) were male and upper limb fractures were more common (84\%). They were assigned to receive 1\% ketamine at a dose of $0.5 \mathrm{mg} / \mathrm{kg}(\mathrm{within} 5 \mathrm{~s})$, and in the control group, a number of 98 patients participated with a mean age of $9.08 \pm 3.98$, among them $73(74.5 \%)$ were male and upper limb fractures were more common (83.7\%). They received ketamine at $1.5 \mathrm{mg} / \mathrm{kg}$ for 30-60 $\mathrm{s}$. The outcomes were measured, and the satisfaction of patients and physicians was recorded.

Results: Successful sedation rate was significantly lower in the low-dose ketamine group than in the control group ( $7 \%$ vs $100 \%$; $<<0.001)$. Moreover, the duration of drug effect and recovery rate were significantly lower in the low-dose ketamine group than in the high-dose ketamine group $(p<0.05)$. In addition, the rates of neurological $(20.4 \%$ vs $5 \%$ ) and physiological $(10.2 \%$ vs $2 \%)$ side effects were significantly higher in the control group than in the intervention group $(p<0.05)$.

Conclusion: The findings of this study did not show that low-dose intravenous ketamine bolus had any beneficial effects in sedating paediatric patients, suggesting that it should not be considered as an accompaniment for standard therapy in short-term pain control.

Keywords: Low-dose intravenous ketamine bolus, fracture, children
\end{abstract}

\section{Introduction}

Fractures constitute $10-25 \%$ of all pediatric trauma. The calculated risk for a boy to sustain a fracture before reaching skeletal maturity is between $42 \%$ and $60 \%$ and for a girls is between $27 \%$ and $40 \%$, respectively $(1,2)$. The peak of incidence of fractures in children occurs at the time of peak height velocity: 11.5 and 13.5 years of age in girls and boys, respectively (3).

Ten to twenty-five percentage of trauma in children cause fractures. The risk of fracture before reaching skeletal maturity is about $42 \%$ to $60 \%$ and for girls is $27 \%$ to $40 \%(1,2)$. The incidence of fractures in children is 11.5 and 13.5 for girls and boys, respectively (3). Non-operative treatment of fractures in children includes: restoration of length, anatomic alignment and immobilization, which need appropriate sedation in order to reduce patient awareness (4). For this purpose, different sedation methods and drugs have been introduced such as midazolam, propofol, fentanyl, and also ketamine, which recently has been added to the list of sedative drugs for fracture (5).

Recently, ketamine has been introduced as a common drug given to sedate children for different painful procedures in the emergency department (ED) (6-8). Ketamine is one of the phencyclidine derivatives with quick onset and short duration of action (9), which is cost-effective, easily accessible, stable at room temperature and has safe sedation and analgesia recommended by the World Health Organization, as an essential medication for 
sedation procedures (10-16). In the study conducted by McCarty et al. (17), it was shown that ketamine is reliable, safe, and provides quick and adequate sedation in children's fractures in ED. Ketamine at doses more than $1 \mathrm{mg} / \mathrm{kg}$ has been routinely used in brief procedures in ED, causing many adverse effects, including neuropsychiatric problems, especially in pediatric patients $(18,19)$, such as hallucinations in the short periods of time $(20,21)$, unpleasant dreams, or acute psychosis (22). To the extent that we know, only a few prospective studies exist regarding the administration of lower doses of ketamine in ED. Moreover, contrary results have been reported for ketamine in sub-anaesthetic doses; therefore, we decided to assess and compare the clinical efficacy of low-dose intravenous ketamine bolus versus usual dosage of ketamine, for reduction of upper and lower extremity fractures in children.

\section{Materials and Methods}

\section{Study Design and Participants}

This prospective double-blind clinical trial was carried out in the ED of Isfahan Al-Zahra University Hospital, from May 2016 to June 2017 (ClinicalTrials.gov Identifier: NCT03499886). The study was approved by the Ethical Committee of Isfahan University of Medical Sciences in April 2016 (registration number: 395779), and all participants and their parents signed the informed consent form. The sedation levels and different outcomes of patients receiving low-dose intravenous ketamine bolus (intervention group) were compared to patients receiving higher doses of intravenous ketamine (control group), for reduction of upper and lower extremity fractures. Inclusion criteria consisted of parents' desire and consent to participate in the study, body mass index within the normal range, having age of 6 months to 17 years, requiring a reduction of upper and lower limb fractures, not receiving benzodiazepines and other sedative drugs before intervention.

Exclusion criteria consisted of patients' age $<3$ months with the body temperature of $>38{ }^{\circ} \mathrm{C}$, due to upper respiratory tract infection. Patients having any other complications such as cardiovascular, gastrointestinal, psychological and neurological were excluded from the study. We also excluded the patients who have withdrawn from the study.

\section{Participants and Intervention}

The study flow diagram is depicted in Figure 1. Two hundred and ten patients with a diagnosis of upper and lower extremity fractures, diagnosed by Emergency Medicine Specialist, were enrolled based on X-ray images and inclusion criteria.

The participants were randomly divided into two groups, using a permuted random allocation program (available at www.graphpad.com/quickcalcs) while maintaining a balance across treatment groups with matching participants in each block based on sex and age. Setting the power of the study at $80 \%$ and at $95 \%$ confidence limit, the calculated sample size becomes two hundred. One hundred ninety-eight patients, however, completed the study; including one hundred from the intervention group and 98 from the control group.

After informed consent was obtained, eligible patients were enrolled. All patients were monitored by direct observation and continuous cardiovascular monitoring, in order to check the vital signs and also by pulse oximeters to examine the blood oxygen saturation level.

Tools and measures used for each patient during the process were as follows:

1- Recurrent suction and, if necessary, use of atropine to control excessive salivation

2- Laryngospasm and respiratory suppression and, airway management measures, in case of any complications

3- Controlling the heart rate and blood pressure, intracranial pressure and performing sedation and using beta-blocker, if needed

4- Forecasting emergency response and providing a quiet and dark room and administrating midazolam, if needed.

In the intervention group, ketamine $1 \%$ was administered rapidly at a dose of $0.5 \mathrm{mg} / \mathrm{kg}$ (within 5 seconds), and in the control group, ketamine $1.5 \mathrm{mg} / \mathrm{kg}$ was slowly injected for 30 to 60 seconds. Patient assessment was performed before and two minutes after ketamine injection, and then every 5 minutes after the reduction of the fracture, by an anaesthetist blind to the type of intervention.

Finally, after the intervention, the patients were put under close and direct observation in a quiet and dim room. The incidence of any side effects of ketamine injections such as gastrointestinal (nausea, vomiting, increase in salivary secretion), neurological (light-headedness, dizziness, headache, visual disturbance, nystagmus, numbness, drowsiness, or increased skeletal tone), psychological (dysphoria or confusion, hallucination, disorientation, mood change or agitation) and cardiopulmonary (major: hypotension and hypoxia; minor: hypertension and tachycardia) were recorded by a nurse, blinded to the intervention group; if necessary, immediate action was taken.

Aldrete scoring system was used to assess the recovery and to determine the patient readiness for discharge. Wisconsin sedation scale and the Aldrete scoring system were calculated for each patient; if the Wisconsin sedation scale was $>5$ and the 
Aldrete scoring system $>10$, the patient was ready to discharge from the ED.

\section{Statistical Analysis}

Only data from patients with complete information were analysed and reported. Statistical analysis of data was carried out by SPSS version 22 software. To compare qualitative variables between groups chi-square test was used. In order to assess the normal distribution of all quantitative studied parameters, Kolmogorov-Smirnov test was used. We used also Student's t-test and paired t-test for normally distributed variables. In addition, for variables without normal distribution, Mann-Whitney $U$ and Wilcoxon tests were used. The two tailed $p$ value less than 0.05 were considered statistically significant (Figure 1).

\section{Results}

Demographic features, in terms of age $(p=0.856)$, sex $(p=0.68)$ were similar in both groups (Table 1). Twelve patients were withdrawn from the study and 198 patients completed the study.
Results showed that the successful sedation rate, in the low dose ketamine group was significantly lower as compared to control group (7\% vs 100\%) $(p<0.001)$. Moreover, in terms of duration of the drug effect and of recovery, the low dose ketamine group were significantly lower compared to the other group, receiving higher doses of ketamine $(p<0.05)$. Furthermore, the sedation depth based on Wisconsin sedation scale was significantly higher in the low dose ketamine group compared to the other group. These data suggest that the sedation level, in patients responded to the drug $(n=7)$, was spontaneous without the stimulus (awake and calm or drowsy with eyes open or closed, easily aroused), while in the control group required sustained painful stimulus (levels: 1-3) $(p<0.001)$. Moreover, patients and Physician satisfaction with control group was significantly higher than low dose ketamine group $(p<0.001)$.

By evaluating different complications, we found that the rate of neurological (20.4\% vs 5\%) and physiological (10.2\% vs $2 \%$ ) adverse events in the control group was meaningfully higher than low dose ketamine group $(p<0.05)$, while the other complications

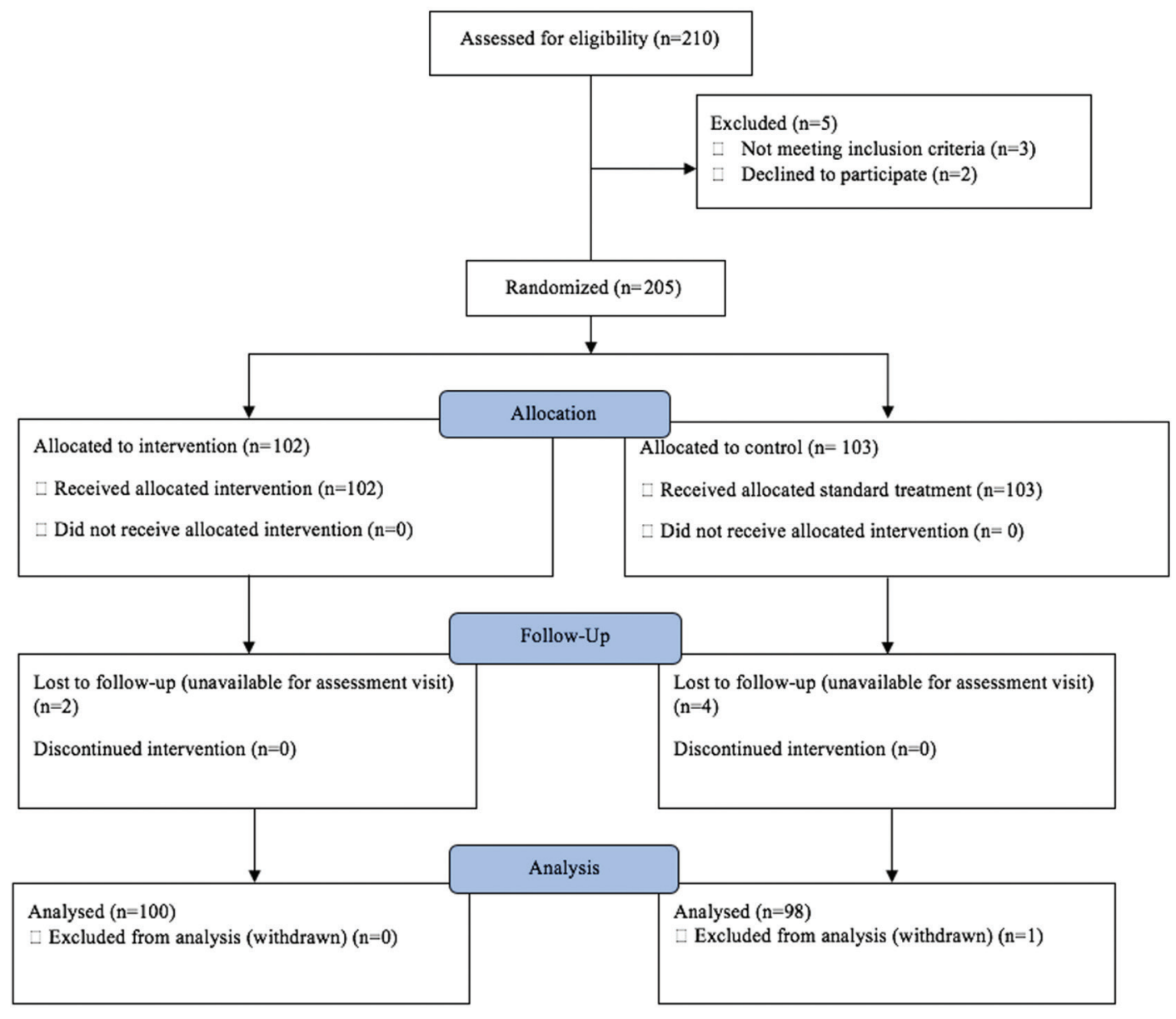

Figure 1. Study flowchart (CONSORT flow diagram)

CONSORT: Consolidated standards of reporting trials, n: Number 


\begin{tabular}{|c|c|c|c|c|}
\hline \multicolumn{2}{|l|}{ Group variables } & Low dose ketamine & Control & p-value \\
\hline \multicolumn{2}{|l|}{ Age (years) } & $8.89 \pm 4.37$ & $9.08 \pm 3.98$ & 0.856 \\
\hline \multirow{2}{*}{$\begin{array}{l}\text { Type of } \\
\text { fracture }\end{array}$} & Upper extremities & $84(84 \%)$ & $82(83.7 \%)$ & \multirow{2}{*}{0.95} \\
\hline & Lower extremities & $16(16 \%)$ & $16(16.3 \%)$ & \\
\hline \multicolumn{2}{|c|}{ Starting the drug effect (s) } & $54.28 \pm 12.72$ & $62.57 \pm 8.61$ & 0.048 \\
\hline \multicolumn{2}{|c|}{ Duration of the drug effect (min) } & $13.42 \pm 6.13$ & $17.61 \pm 4.68$ & 0.034 \\
\hline \multicolumn{2}{|c|}{ Duration of recovery (min) } & $15.28 \pm 4.15$ & $65 \pm 12.69$ & $<0.001$ \\
\hline \multicolumn{2}{|c|}{ Wisconsin sedation scale } & $5.53 \pm 0.74$ & $1.95 \pm 0.71$ & $<0.001$ \\
\hline \multicolumn{2}{|c|}{ Patients satisfaction } & $1.17 \pm 0.49$ & $4.58 \pm 0.53$ & $<0.001$ \\
\hline \multirow{4}{*}{ Complications } & Neurological & $5(5 \%)$ & $20(20.4 \%)$ & 0.001 \\
\hline & Physiological & $2(2 \%)$ & $10(10.2 \%)$ & 0.016 \\
\hline & Minor cardiopulmonary & $1(1 \%)$ & $6(6.1 \%)$ & 0.064 \\
\hline & Major cardiopulmonary & 0 & 0 & - \\
\hline
\end{tabular}

such as gastrointestinal and cardiopulmonary (minor and major) adverse events did not show any differences among the groups $(p>0.05)$.

\section{Discussion}

Sedation with intravenous ketamine in children conventionally involves doses of 1 to $2 \mathrm{mg} / \mathrm{kg}$, administered slowly. The main objective of this study was to demonstrate if lower doses of ketamine, pushed rapidly achieve adequate sedation. Our data show, prescribing low dose ketamine $(0.5 \mathrm{mg} / \mathrm{kg})$ for reduction of fractures in children, did not induce proper sedation levels, while by increasing the dosage to $1.5 \mathrm{mg} / \mathrm{kg}$, appropriate sedation was achieved in the ED; however, the side effects especially neurological and physiological complications were increased in the latter dosage.

In 2012, a study arranged by Yazigi et al. (23), found that low-dose ketamine, did not decrease acute pain scores and supplemental morphine was required following thoracotomy, even in combination with a continuous intercostal nerve block. Furthermore, they found that the rate of complications such as psychomimetic adverse effects did not differ as compared to the control group. Edwards et al. (24) reported that a combination of morphine and low-doses ketamine in elderly patients, after upper abdominal surgery did not significantly decrease the pain and did not improve the lung function. Another study performed by Menigaux et al. (25) showed that low-dose ketamine $(0.15$ $\mathrm{mg} / \mathrm{kg}$ ) had no beneficial effect on pain scores at rest, following orthopaedic surgery. However, in terms of studied population, sample size and chief complaints were different compared to ours, but the results were consistent with our data.

While in 2016, a study performed by Lee and Lee (26) showed that the effect of ketamine on pain reduction differs according to the pain site. Furthermore, they demonstrated that low-dose ketamine may be a key modality for pain management in ED, without causing any side effects. Ahern et al. (27) showed that low-dose ketamine infusion in ED resulted in significant pain control, with generally mild adverse events and with no severe side effects such as major cardiopulmonary complications. Goltser et al. (28) demonstrated that low-dose ketamine infusion may be safe and effective drug in pain reduction for patients with a history of chronic opioid use. Moreover, the results of Ahmadi et al. (29) in 2014, showed that low-dose ketamine in accompany with midazolam has an analgesic effect similar to morphine, on pain relief in closed limb fractures, with less respiratory adverse events. Furthermore, Pandit et al. (30) found that lowdose intravenous ketamine bolus, produced marked sedation for about 20 minutes, followed by moderate sedation during intravenous infusion in patients in ED.

The results of these studies were in contrast to our study, which may be due in part to differences in sample size, race, demographic features (children age groups) and chief complaints. Furthermore, we evaluated seven patients in low- 
dose intravenous ketamine group, which in regards to small sample size, it is very small for evaluating the other outcomes such as the sedation levels, satisfaction and complications. Therefore, further studies are required to use higher sample size with different demographic features, in order to find the exact effect of low-dose intravenous ketamine bolus injection. As the rate of adverse events in this group was significantly low, appropriate patient sample size is required in order to examine the response to the indicated dosage.

\section{Conclusion}

The findings of this study did not show any beneficial effects of low-dose intravenous ketamine bolus in sedating paediatric patients, for reduction of upper and lower extremity fractures. Our data suggest that the indicated low dose should not be prescribed as a substitute for standard therapy and control of pain in the short term; however, side effects were noted to be significantly lower.

\section{Acknowledgments}

This study was financially supported by the Technology and Research Development Department of Isfahan University of Medical Sciences. We gratefully appreciate the dedicated efforts of the researchers, the coordinators and the volunteer patients who contributed to this study, and the Clinical Research and Development Units (CRDU) of Al-Zahra University.

\section{Ethics}

Ethics Committee Approval: This article is approved by Ethics Committee of Isfahan University of Medical Sciences. Ethic approval number is 395779 .

Informed Consent: All participants and their parents signed the informed consent form after informed consent was obtained.

Peer-review: Externally peer-reviewed.

\section{Authorship Contributions}

Surgical and Medical Practices: M.N.I., N.S., K.G., Concept: M.N.I., Design: M.N.I., N.S., Data Collection or Processing: M.N.I., N.S., K.G., Analysis or Interpretation: M.N.I., N.S., K.G., Literature Search: M.N.I., N.S., K.G., Writing: M.N.I., N.S., K.G.

Conflict of Interest: The authors declare no conflicts of interests regarding the content of this article.

Financial Disclosure: The authors declared that this study received no financial support.

\section{References}

1. Landin LA. Epidemiology of children's fractures. J Pediatr Orthop B. 1997:6:79-83.
2. Karlsson MK, Duan Y, Ahlborg H, Obrant KJ, Johnell O, Seeman E. Age, gender, and fragility fractures are associated with differences in quantitative ultrasound independent of bone mineral density. Bone. 2001;28:118-22.

3. Tanner JM, Davies PS. Clinical longitudinal standards for height and height velocity for North American children. J Pediatr. 1985;107:317-29.

4. Constantine E, Steele DW, Eberson C, Boutis K, Amanullah S, Linakis JG. The use of local anesthetic techniques for closed forearm fracture reduction in children: a survey of academic pediatric emergency departments. Pediatr Emerg Care. 2007;23:209-11.

5. Migita RT, Klein EJ, Garrison MM. Sedation and analgesia for pediatric fracture reduction in the emergency department: a systematic review. Arch Pediatr Adolesc Med. 2006;160:46-51.

6. Godambe SA, Elliot V, Matheny D, Pershad J. Comparison of propofol/fentanyl versus ketamine/midazolam for brief orthopedic procedural sedation in a pediatric emergency department. Pediatrics. 2003;112:116-23.

7. Luhmann JD, Schootman M, Luhmann SJ, Kennedy RM. A randomized comparison of nitrous oxide plus hematoma block versus ketamine plus midazolam for emergency department forearm fracture reduction in children. Pediatrics. 2006;118:e1078-86.

8. Green SM, Roback MG, Kennedy RM, Krauss B. Clinical practice guideline for emergency department ketamine dissociative sedation: 2011 update. Ann Emerg Med. 2011;57:449-61.

9. Morrison T, Carender C, Kilbane B, Liu RW. Procedural Sedation with Ketamine Versus Propofol for Closed Reduction of Pediatric Both Bone Forearm Fractures. Orthopedics. 2017;6:1-7. [Epub ahead of print].

10. World Health Organisation. WHO Model List of Essential Medicines for Children, 5 th List (April 2015) WHO Medicines. https://www.who.int/ selection_medicines/committees/expert/20/CORRIGENDA_Rev.1_EMLC_ BC.pdf?ua=1 (accessed 8 Feb 2016).

11. Donnelly RF. Stability of diluted ketamine packaged in glass vials. Can J Hosp Pharm. 2013;66:198.

12. Walker SE, Law S, DeAngelis C. Stability and compatability of hydromorphone and ketamine in normal saline. CJHP. 2001:193-201.

13. Kohrs R, Durieux ME. Ketamine: teaching an old drug new trick. Anesth Analg. 1998;87:1186-93.

14. Reich DL, Silvay G. Ketamine: an update on the first twenty-five years of clinical experience. Can J Anaesth. 1989;36:186-97.

15. Smith DC, Mader TJ, Smithline HA. Low dose intravenous ketamine as an analgesic: a pilot study using an experimental model of acute pain. Am J Emerg Med. 2001;19:531-2.

16. Nasr A, Alimohammadi N, Isfahani MN, Alijanpour S. Development and Domestication of a Clinical Guideline for Pharmacological Pain Management of Trauma Patients in Prehospital Setting. ATR. 2019;8:111.

17. McCarty EC, Mencio GA, Walker LA, Green NE. Ketamine sedation for the reduction of children's fractures in the emergency department. J Bone Joint Surg Am. 2000;82-A:912-8.

18. Weinbroum AA. A single small dose of postoperative ketamine provides rapid and sustained improvement in morphine analgesia in the presence of morphine-resistant pain. Anesth Analg. 2003;96:789-95.

19. Nesher N, Serovian I, Marouani N, Chazan S, Weinbroum AA. Ketamine spares morphine consumption after transthoracic lung and heart surgery without adverse hemodynamic effects. Pharmacol Res. 2008;58:38-44.

20. Hartvig P, Larsson E, Joachimsson PO. Postoperative analgesia and sedation following pediatric cardiac surgery using a constant infusion of ketamine. J Cardiothorac Vasc Anesth. 1993;7:148-53.

21. Green SM, Nakamura R, Johnson NE. Ketamine sedation for pediatric procedures: Part 1, A prospective series. Ann Emerg Med. 1990;19:1024-32.

22. Malhotra AK, Pinals DA, Weingartner $\mathrm{H}$, Sirocco K, Missar CD, Pickar D, et al. NMDA receptor function and human cognition: The effects of ketamine in healthy volunteers. Neuropsychopharmacol. 1996;14:301-7. 
23. Yazigi A, Abou-Zeid H, Srouji T, Madi-Jebara S, Haddad F, Jabbour K. The effect of low-dose intravenous ketamine on continuous intercostal analgesia following thoracotomy. Ann Card Anaesth. 2012;15:32-8.

24. Edwards ND, Fletcher A, Cole JR, Peacock JE. Combined infusions of morphine and ketamine for postoperative pain in elderly patients. Anaesthesia. 1993;48:124-7.

25. Menigaux C, Fletcher D, Dupont X, Guignard B, Guirimand F, Chauvin M. The benefits of intraoperative small-dose ketamine on postoperative pain after anterior cruciate ligament repair. Anesth Analg. 2000;90:129-35.

26. Lee EN, Lee JH. The Effects of Low-Dose Ketamine on Acute Pain in an Emergency Setting: A Systematic Reviewand Meta-Analysis. PLoS One. 2016;11:e0165461.
27. Ahern TL, Herring AA, Miller S, Frazee BW. Low-Dose Ketamine Infusion for Emergency Department Patients with Severe Pain. Pain Med. 2015;16:14029. [Epub 2015 Feb 3].

28. Goltser A, Soleyman-Zomalan E, Kresch F, Motov S. Short (low-dose) ketamine infusion for managing acute pain in the ED: case-report series. Am J Emerg Med. 2015;33:601.e5-7. [Epub 2014 Sep 26].

29. Ahmadi O, Isfahani MN, Feizi A. Comparing low-dose intravenous ketaminemidazolam with intravenous morphine with respect to pain control in patients with closed limb fracture. J Res Med Sci. 2014;19:502-8.

30. Pandit SK, Kothary SP, Kumar SM. Low dose intravenous infusion technique with ketamine. Amnesic, analgesic and sedative effects in human volunteers. Anaesthesia. 1980;35:669-75. 